\title{
Prospettive terapeutiche nella cura del morbo di Addison
}

\section{Duccio Lombardi}

Dottorando in Farmacologia Preclinica e Clinica presso il Laboratorio Interdipartimentale di Nefrologia Cellulare e Molecolare, Centro DENOTHE, Univesità degli Studi di Firenze, Firenze

In collaborazione con AIPAd (Associazione Italiana Pazienti Addison)

Therapeutic Perspectives in the TREatment of Addison's disease

Abstract. Innovative therapeutic applications that could lead to a new and different approach for the treatment of Addison's disease are considered and evaluated in this editorial. Even though the information on Addison's disease is still limited, mostly due to its rarity, the main pathogenetic mechanisms of the disease are here discussed as to explore potential future strategies for the treatment of this pathology. This editorial analyzes the genetic and immunological factors in Addison's disease, and tries to understand which could be the role of the adrenocortical stem cells for the regeneration of the glandular functions. At the end, after two clinical trials are pointed out, the editorial puts forward the hypothesis of some therapeutic possibilities that could open new perspectives on how to treat patients affected by this autoimmune disease.

Key words: Addison's disease, Cellular therapies, Stem cells, New therapeutic possibilities

Conflict of interest: None.

Financial support: None.

Ricevuto: 16 Dicembre 2012; Accettato: 1 Febbraio 2013

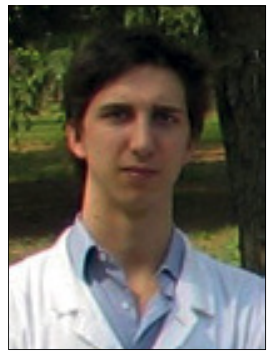

Duccio Lombardi

\section{Introduzione}

Le prospettive terapeutiche indirizzate alla cura del morbo di Addison (AD) sono influenzate e limitate da una delle caratteristiche chiave di questa patologia. Infatti, l'AD è una condizione autoimmune endocrina rara, che colpisce circa 100 persone per ogni milione di individui nella popolazione di origine caucasica (1). I primi e più importanti fattori da considerare nell'analisi delle possibilità terapeutiche relative al morbo di Addison sono: a) la sua bassa prevalenza, b) la scarsa conoscenza del compartimento staminale surrenale (intrinsecamente adibito al mantenimento dell'omeostasi cellulare dell'organo) e c) la scarsa conoscenza degli eventi autoimmuni scatenanti la malattia.

Circa il 70-75\% dei casi di morbo di Addison è dovuto a un'aggressione autoimmune della ghiandola surrenale. In particolare, la patogenesi del morbo di Addison prevede che, nella regione corticale surrenalica, antigeni che normalmente non innescano un'attivazione della risposta immunitaria grazie all'intervento di meccanismi di tolleranza immunologica, diventino obiettivo dell'attacco da parte del sistema immunitario. Per questo motivo, in circa l' $^{\prime} 85 \%$ dei pazienti affetti da $\mathrm{AD}$, si possono rilevare autoanticorpi diretti verso la 21 -idros- silasi (2) o verso altri importanti enzimi sempre coinvolti nella steroidogenesi, come la 17- $\alpha$-idrossilasi.

Che una risposta immunologica sia alla base della forma autoimmune di AD e che la caratterizzi è sicuramente sostenuto dalla presenza di un infiltrato linfo-monocitario riscontrabile a livello della corticale surrenalica. È importante ricordare che la presenza di autoanticorpi e di infiltrato infiammatorio sono strettamente correlati tra loro e aumentano proporzionalmente della stessa intensità, mentre la steroidogenesi, indice di funzionalità surrenalica, decresce in modo inversamente proporzionale all'aumentare di questi due fattori. Si è, quindi, di fronte a una sorta di circolo vizioso che conduce a una sempre minor produzione di steroidi, evento che porta, infine, allo sviluppo della patologia conclamata (1).

Gli autoanticorpi responsabili della patogenesi dell'AD, chiamati anche ACA (Anti-Cortex Antibodies), sono molto specifici e occasionalmente si possono trovare anche in pazienti affetti da morbo di Basedow o da tiroidite di Hashimoto. La loro presenza correla con il rischio di sviluppo di insufficienza surrenalica e, soprattutto, può essere riscontrata anche molti anni prima rispetto alla comparsa della sintomatologia clinica associata all'AD (3).

È comunque stato descritto che non tutti gli individui in cui siano stati riscontrati tali ACA abbiano poi sviluppato la patologia. Addirittura, in alcuni casi, è stata osservata una regressione nella presenza di autoanticorpi sino a uno stato di assen- 
za degli stessi (4), il che potrebbe essere dovuto a un innesco di sistemi periferici di tolleranza come anergia, delezione o soppressione immunitaria.

Come avviene per la grande maggioranza di patologie di natura autoimmune, il morbo di Addison è spesso associato ad altri tipi di autoimmunità, i quali possono presentarsi anticipatamente o successivamente rispetto all'insorgenza dell'AD. E quindi chiaro che i pazienti possono presentare sia un'AD isolata, che un' $\mathrm{AD}$ che rientra come parte di una sindrome da poliendocrinopatia autoimmune (o SPA), la quale può manifestarsi in due forme.

La prima forma, o SPA1, è una patologia monogenica e autosomica recessiva dovuta a una mutazione del gene autoimmune regulator (AIRE). Questo gene è ampiamente noto per essere coinvolto nella tolleranza centrale che si verifica a livello timico sui Linfociti $\mathrm{T}$. In tale sede sono eliminati per selezione negativa i Linfociti $\mathrm{T}$ in grado di riconoscere antigeni autologhi, i quali sono qui espressi dalle cellule epiteliali della midollare grazie all'induzione della trascrizione mediata proprio da AIRE (5). La SPA1 è particolarmente rara e si manifesta spesso in giovane età con candidiasi muco-cutanee croniche, insufficienza surrenalica e ipoparatiroidismo autoimmune; inoltre, può progredire con l'instaurarsi di nuovi disordini autoimmuni come il diabete mellito di tipo 1 o l'anemia perniciosa (5).

La SPA2 è, invece, identificabile come un'AD associata a disfunzioni tiroidee di natura autoimmune o a diabete mellito di tipo 1; essa presenta, inoltre, un'incidenza di circa il $50 \%$ in individui affetti da un'insufficienza surrenalica primaria (6).

\section{La genetica del Morbo di Addison}

Da molto tempo l'AD è stato riconosciuto come un disordine di origine ereditaria (1). L'investigazione delle basi genetiche di una patologia già così rara e complessa è però ulteriormente complicata dal fatto che presenta una penetranza incompleta e che un ampio numero di fattori ambientali è potenzialmente coinvolto nella sua eziopatogenesi.

Tramite modelli sperimentali animali di AD è stato possibile individuare come "hot spots" le regioni geniche MHC e CTLA-4 (7); purtroppo, però, la rarità dell'incidenza di questa patologia nell'uomo non ha ancora permesso di condurre analisi genetiche su numeri di casi sufficienti che permettano di giungere a conclusioni più stringenti. Tali investigazioni si rivelerebbero particolarmente utili data la complessa eziologia di una patologia che richiede l'interazione tra svariate varianti geniche e fattori ambientali ancora non del tutto noti.

Gli studi condotti fino a questo momento hanno comunque permesso di definire una linea generale di pensiero sull'influenza della genetica nell'insorgenza della patologia. In particolare, è stato descritto che la maggior parte dei loci definiti come parzialmente responsabili del morbo di Addison codifica fattori che influenzano vie di segnalazione immunologiche, inerenti soprattutto all'immunità adattiva (1).

Mutazioni nel locus MHC possono portare ad alterazioni nelle proteine di membrana HLA, molecole coinvolte nella presentazione dell'antigene ai Linfociti $\mathrm{T}$ e alla loro attivazione.

In particolare, va sottolineato come varianti dei geni MHC di classe II (codificanti per HLA espresse unicamente cellule presentanti l'antigene, o APC) siano fortemente associate a varie condizioni di autoimmunità (7). Ciò è molto probabilmente dovuto al fatto che alcuni polimorfismi MHC codificano per varianti proteiche HLA che permettono la presentazione di alcuni autoantigeni, i quali possono, quindi, essere presentati a Linfociti $\mathrm{T}$ specifici che ne risulteranno attivati innescando una risposta immune, come si suppone avvenire per la 21-idrossilasi.

Altro ruolo di primo piano è giocato dalle molecole co-stimolatorie. Fisiologicamente queste molecole sono richieste per completare i segnali necessari per un'attivazione e una risposta adeguate del Linfocita $\mathrm{T}$ allo stimolo da parte dell'antigene.

Un esempio su tanti è CTLA4, gene che codifica per una molecola co-stimolatoria che controlla in senso negativo le risposte del Linfocita $T$, inducendo questo verso uno stato di non responsività qualora riconosca autoantigeni (8). Varianti geniche di tale locus sono coinvolte nell'eziologia di numerose patologie autoimmuni, come nel caso di diabete insulinodipendente o nella tiroidite di Hashimoto (1). Se tale gene non fosse funzionale nel prevenire l'attivazione erronea dei Linfociti $T$, è plausibile che Linfociti autoreattivi possano essere innescati anche in caso di presentazione di autoantigeni ad opera delle APC, avviando, così, la patologia autoimmune. È inoltre interessante notare come individui affetti da $\mathrm{AD}$ presentino ridotti livelli della forma secreta e solubile di CTLA4, evento che permette alle cellule presentanti l'antigene di stimolare e attivare con maggiore frequenza Linfociti T potenzialmente autoreattivi (1).

\section{Le Cellule Staminali Surrenaliche}

Le cellule staminali adrenocorticali sono state identificate solo recentemente, anche se la loro presenza era già stata ipotizzata da tempo. Tali cellule risiedono nella periferia della regione corticale surrenalica e sono un'entità indifferenziata e quiescente che inizia, però, a proliferare e a differenziare terminalmente non appena insorge la necessità di rimpiazzare cellule morte.

Per tali cellule staminali un ruolo di primo piano è rivestito dal gene Sf1 (Steroidogenic Factor 1), regolatore trascrizionale di geni codificanti enzimi deputati alla sintesi di ormoni steroidei. La sua espressione non solo è critica per le funzioni steroidogeniche sia durante lo sviluppo fetale che in età adulta, ma è anche essenziale per la corretta organogenesi delle ghiandole surrenali. Infatti, in modelli murini mancanti del gene Sfl, tali organi non si formano (9).

Una delle ipotesi a oggi di maggior rilievo è che nella regione capsulare sia localizzata la nicchia staminale surrenalica, mentre nella regione sottostante la capsula sarebbero riscontrabili cellule transizionali, derivanti dalla stessa regione capsulare e pronte a differenziare per sostituire le cellule perse $(9,10)$. Ma se un primo problema a un approccio terapeutico basato su una terapia cellulare è rappresentato dalla scarsa conoscenza del compartimento staminale surrenalico, un fattore aggiuntivo non trascurabile limita le applicazioni basate su cellule staminali nel trattamento del morbo di Addison. L'AD 
è, infatti, una patologia autoimmune, perciò, senza un previo blocco delle reazioni che danno luogo all'autoimmunità, è impossibile pensare ad approcci basati su qualsiasi tipo di entità staminale. Se, infatti, dovessimo ipotizzare l'impiego di terapie cellulari volte a sostituire le cellule steroidogeniche perse, senza però prima fermare le reazioni di autoimmunità, il risultato sarebbe quello di continuare a fornire substrati che alimenterebbero il "fuoco" dell'autoimmunità stessa.

Tuttavia, è possibile ipotizzare l'impiego di cellule staminali di varia natura, in seguito al blocco della reazione autoimmune, per la ricostruzione dell'architettura e della funzionalità delle ghiandole surrenali, nonché per il ripristino della sintesi di ormoni steroidei.

\section{Possibilità Terapeutiche}

Attualmente, il morbo di Addison è trattato mediante terapia sostituiva orale a base di idrocortisone, prednisone o prednisolone, molecole che vanno a sostituire il cortisolo non più prodotto dalle ghiandole surrenali. I regimi terapeutici hanno come finalità quella di mimare le concentrazioni fisiologiche di cortisolo, in relazione sia al ritmo circadiano, che a varie condizioni come sforzi fisici o stress emotivi. Questa è una terapia che dovrà essere mantenuta per tutta la vita del paziente e che molto spesso dovrà essere integrata con fludrocortisone acetato come sostituto dell'aldosterone.

Sebbene questo trattamento sostituivo sia efficace, esso presenta dei limiti intrinseci. Il più evidente è sicuramente quello di dare luogo a concentrazioni di cortisolo analoghe ma non identiche a quelle che fisiologicamente si andrebbero a rilevare, per esempio, in seguito a un dato sforzo, eseguito a una data ora della giornata. A questo si deve aggiungere il costante rischio di episodi acuti che possono richiedere trattamenti di emergenza per via endovenosa.

Prima di analizzare brevemente le prospettive terapeutiche per i pazienti affetti da morbo di Addison è, quindi, necessario richiamare l'importanza di una diagnosi non solo tempestiva, ma, soprattutto, precoce. Condizioni quali la familiarità o l'insorgenza di altre patologie autoimmuni spesso correlate all'AD dovrebbero spingere l'individuo a effettuare la ricerca degli ACA ben prima dell'insorgenza delle manifestazioni cliniche. Questo potrebbe permettere, per esempio, di attuare una terapia immunosoppressiva volta a interferire con i meccanismi attivatori ed effettori delle cellule del sistema immunitario, tra cui quelle autoreattive. Ma le terapie immunosoppressive alle quali si fa più comunemente ricorso in ambito clinico presentano la problematica di essere perlopiù aspecifiche: esse non sono, infatti, in grado di colpire univocamente le cellule autoreattive.

Di maggiore impatto potrebbero invece essere le terapie immunosoppressive su base cellulare. Tra queste spiccano sicuramente quelle basate sull'impiego di cellule staminali mesenchimali (MSC), le quali sono in grado di modulare l'attività del sistema immunitario (11). Queste cellule sono riscontrabili nel midollo osseo, nel tessuto adiposo, nella milza, nel timo e, in basse concentrazioni, anche nel sangue periferico. Le MSC presentano, inoltre, una notevole plasticità differenziativa: esse possono dare luogo a cellule del tessuto adiposo, osseo, cartilagineo e persino muscolare e nervoso, sotto appropriati stimoli.

In particolare, è stato osservato che Linfociti $\mathrm{T}$ attivati da un dato antigene, se coltivati con tale antigene in presenza di $\mathrm{MSC}$, non proliferano. Ciò si pensa sia dovuto alla capacità delle cellule staminali mesenchimali di produrre molecole di carattere immunosoppressivo (TGF $\beta 1$ solubile, CTLA4 e IDO) in grado di bloccare l'attivazione dei Linfociti T (12). Le MSC sono infatti sempre più studiate per tali loro caratteristiche immunomodulatorie, le quali le rendono delle ottime candidate per l'impiego clinico nell'ambito di varie patologie, come in caso di malattia del trapianto contro l'ospite (Graft versus Host Disease, GVHD).

Oltre alle MSC, esistono delle particolari popolazioni linfocitarie in grado di controllare l'attività ed eventualmente di bloccare i Linfociti T effettori. Queste popolazioni "regolatrici” sono i cosiddetti Linfociti T regolatori (Treg). Le cellule Treg offrono grandi speranze da un punto di vista applicativo, in quanto possono essere derivate dal singolo soggetto e permettono un'immunosoppressione altamente specifica e diretta univocamente verso le cellule di interesse, in questo caso le cellule autoreattive.

Le Treg, che possono essere espanse in vitro in presenza di antigene e di cellule presentanti l'antigene, sono in grado di sopprimere l'attivazione di Linfociti $\mathrm{T}$ effettori diretti verso lo stesso antigene che è stato loro presentato dalle APC (13, 14). Se, quindi, si possono creare Treg antigene-specifiche, è plausibile ipotizzare di poter realizzare linee cellulari che siano in grado di bloccare specificamente Linfociti $\mathrm{T}$ reattivi nei confronti di cellule esprimenti la 21-idrossilasi o la 17- $\alpha$-idrossilasi.

I Linfociti $\mathrm{T}$ regolatori potrebbero quindi essere una buona nonché interessante risorsa per il trattamento dell'AD, soprattutto in quanto tutti i passaggi precedentemente accennati coinvolgerebbero unicamente cellule autologhe, evitando così il rischio di rigetto immunitario.

Per quanto concerne invece sperimentazioni cliniche in atto, sono da rilevare due trial clinici attualmente in corso e decisamente innovativi rispetto alla classica terapia sostitutiva.

Il primo si chiama "Revival of Stem Cells in Addison's Study" della Newcastle University ( ${ }^{\circ}$ identificativo: NCT01371526), il cui obiettivo è quello di ripristinare le funzioni steroidogeniche in pazienti con AD conclamata mediante stimolazione delle cellule staminali surrenaliche tramite ACTH. Il protocollo di questo studio prevede la somministrazione quotidiana sottocutanea di ACTH per 20 settimane al fine di valutare se avvenga effettivamente la rigenerazione delle funzioni steroidogeniche e se questa sia effettivamente dovuta all'attivazione dei progenitori staminali.

Il secondo trial è denominato invece "Rescue of Steroidogenic Capacity in Adrenocortical Failure Study" ed è sempre condotto dalla Newcastle University ( ${ }^{\circ}$ identificativo: NCT00753597). In questo studio pilota è prevista la deplezione dei Linfociti B, responsabili della produzione di anticorpi, al fine di tentare di salvaguardare la capacità steroidogenica delle ghiandole surrenali in soggetti affetti da morbo di Addison a uno stadio iniziale. Durante le prime 12 settimane di trattamento è somministrata anche la terapia sostitutiva, la 
quale è poi gradualmente diminuita fino a essere interrotta. La funzione surrenalica è quindi rivalutata dopo $13,26,39$ e 52 settimane. L'endpoint primario dello studio è quello di restaurare la funzione steroidogenica: la deplezione dei Linfociti B potrebbe infatti ridurre l'entità dell'attacco immunologico nei confronti delle ghiandole surrenali, permettendo potenzialmente il recupero di uno stato di tolleranza immunologica e di capacità steroidogenica.

Se, quindi, la strada per una cura che permetta il blocco definitivo del morbo di Addison e, in seguito, il ripristino delle funzioni steroidogeniche e dell'architettura delle ghiandole surrenali appare lunga e di non semplice percorrenza, molte sono le nuove applicazioni e le nuove conoscenze del funzionamento biologico del nostro organismo che emergono a ritmo incessante. Per quanto queste applicazioni molto spesso non siano finalizzate al trattamento dell'AD, i fini principi biologici comuni che regolano ogni "compartimento" del nostro corpo permetteranno che ciò che un giorno sarà scoperto per essere applicato in un altro campo, automaticamente potrà diventare adottabile anche per l'Addison.

\section{Ringraziamenti}

Si ringraziano il Professore Francesco Annunziato e il Professore Alessandro Peri per la revisione critica del manoscritto.

\section{Riassunto}

In questo editoriale sono valutate e analizzate alcune applicazioni terapeutiche innovative che potrebbero permet- tere un nuovo e differente approccio alla cura del morbo di Addison. Sebbene le conoscenze relative a questa malattia siano ancora limitate, soprattutto a causa della sua rarità, i principali meccanismi patogenetici del morbo sono qui valutati nell'ottica di capire quali potrebbero essere le future strategie terapeutiche per la cura di questa patologia. Attraverso l'analisi delle questioni di natura genetica e immunologica che caratterizzano l'Addison e cercando di comprendere quale potrebbe essere il ruolo delle cellule staminali adrenocorticali nella rigenerazione delle funzioni ghiandolari, sono, infine, riportati due studi clinici e alcune possibilità terapeutiche che potrebbero cambiare profondamente l'approccio a tale patologia.

Parole chiave: Morbo di Addison, Terapie cellulari, Cellule staminali, Nuove possibilità terapeutiche

Dichiarazione di conflitto di interessi: L'Autore dichiara di non avere conflitto di interessi.

Contributi economici agli Autori: L'Autore dichiara di non aver ricevuto sponsorizzazioni economiche per la preparazione dell'articolo.

\section{Indirizzo degli Autori:}

Dr. Duccio Lombardi

Centro Denothe

Università degli Studi di Firenze

V.le G. Pieraccini 6

50139 Firenze

lombarduccio@alice.it

\section{Bibliografia}

1. Mitchell AL, Pearce SH. Autoimmune Addison disease: pathophysiology and genetic complexity. Nat Rev Endocrinol 2012; 8 (5): 306-16.

2. Winqvist O, Karlsson FA, Kämpe O. 21-Hydroxylase, a major autoantigen in idiopathic Addison's disease. Lancet 1992; 339 (8809): 1559-62.

3. Brozzetti A, Marzotti S, La Torre D, et al. Autoantibody responses in autoimmune ovarian insufficiency and in Addison's disease are IgG1 dominated and suggest a predominant, but not exclusive, Th1 type of response. Eur J Endocrinol 2010; 163 (2): 309-17.

4. Betterle C, Scalici C, Presotto F, et al. The natural history of adrenal function in autoimmune patients with adrenal autoantibodies. J Endocrinol 1988; 117 (3): 467-75.

5. Metzger TC, Anderson MS. Control of central and peripheral tolerance by Aire. Immunol Rev 2011; 241 (1): 89-103.

6. Betterle C, Volpato M, Greggio AN, Presotto F. Type 2 polyglandular autoimmune disease (Schmidt's syndrome). J Pediatr Endocrinol Metab 1996; 9 (Suppl. 1); 113-23.

7. Chase K, Sargan D, Miller K, Ostrander EA, Lark KG. Understanding the genetics of autoimmune disease: two loci that regulate late onset Addison's disease in Portuguese Water Dogs. Int J Immuno- genet 2006; 33 (3): 179-84.

8. Brunet JF, Denizot F, Luciani MF, et al. A new member of the immunoglobulin superfamily--CTLA-4. Nature 1987; 328 (6127): 267-70.

9. Luo X, Ikeda Y, Parker KL. A cell-specific nuclear receptor is essential for adrenal and gonadal development and sexual differentiation. Cell 1994; 77: 481-90.

10. Ching S, Vilain E. Targeted disruption of sonic hedgehog in the mouse adrenal leads to adrenocortical hypoplasia. Genesis 2009; 47: 628-37.

11. Bartholomew A, Sturgeon C, Siatskas M, et al. Mesenchymal stem cells suppress lymphocyte proliferation in vitro and prolong skin graft survival in vivo. Exp Hematol 2002; 30 (1): 42-8.

12. Krampera M, Cosmi L, Angeli R, et al. Role for interferon-gamma in the immunomodulatory activity of human bone marrow mesenchymal stem cells. Stem Cells 2006; 24 (2): 386-98.

13. Cosmi L, Liotta F, Lazzeri E, et al. Human CD8+CD25+ thymocytes share phenotypic and functional features with $\mathrm{CD} 4+\mathrm{CD} 25+$ regulatory thymocytes. Blood 2003; 102 (12): 4107-14.

14. Maggi L, Santarlasci V, Liotta F, et al. Demonstration of circulating allergen-specific CD4+CD25highFoxp3+ T-regulatory cells in both nonatopic and atopic individuals. J Allergy Clin Immunol 2007; 120 (2): 429-36. 